\title{
Neoplasias malignas de laringe em 2019 no estado do Rio de Janeiro: análise epidemiológica
}

\section{Malignant laryngeal neoplasms in 2019 in state of Rio de Janeiro: epidemiological analysis}

\author{
Ana Zeile Melo Rolim ${ }^{1 *}$, Ana Silvia Menezes Bastos²
}

Como citar esse artigo. Rolim, A. Z. M;

Bastos, A. S. M. Neoplasias malignas de

laringe em 2019 no estado do Rio de Janeiro: análise epidemiológica. Revista de Saúde2021 Ago/Nov.; 12 (3): 66-68

\begin{abstract}
Resumo
As neoplasias malignas de laringe são um dos principais tipos de carcinomas de cabeça e pescoço. O diagnóstico é feito por meio da laringoscopia com biopsia. O principal tipo é o carcinoma de células escamosas e precisa ser diagnosticado previamente, tendo em vista a sua importância anatômica na deglutição, respiração e fonação - além de sua relevância estética. O objetivo desta análise é descrever o cenário epidemiológico das neoplasias malignas de laringe no estado do Rio de Janeiro, no ano de 2019, a partir da relação de dados importantes, como o número de internações e a taxa de mortalidade dos pacientes acometidos. Esses dados foram coletados por meio de levantamento feito com uso das informações disponíveis no DATASUS e na literatura qualitativa. Foram obtidas respostas de que se trata de uma enfermidade de diagnóstico tardio, que afetou profundamente a vida social de pacientes. Foram identificados 610 casos totais. Considerando o sexo masculino, identificaram-se 518 casos. A raça predominante é a branca. Duas características relevantes desse grupo foram o etilismo e o tabagismo. Diante disso, o diagnóstico precoce é de suma importância para garantir a qualidade de vida do paciente, assim como reduzir o tempo e os gastos com o tratamento que inclui cirurgia e radioterapia.
\end{abstract}

Palavras-chave: Laringe; Neoplasia; Epidemiológico.

\begin{abstract}
Malignant neoplasms of larynx are one of the main types of head and neck carcinomas and the diagnosis is made by laryngoscopy with biopsy. The main type is the squamous cell carcinoma and needs to be diagnosed beforehand, considering that it's anatomical importance in swallowing, breathing and phonation, in addition to aesthetics. The purpose of this analysis is to describe the epidemiological scenario of malignant laryngeal neoplasm in the state of Rio de Janeiro in 2019 , relating important data such as number of hospitalizations mortality rate to the profile of affected patients and these data were collected though a survey made though information available in DATASUS and qualitative literature. Answers were that it is a disease with a late diagnosis which profoundly affected the social life of pacients in 2019.610 total cases were identified. Considering the male gender 518 cases were identified. The predominant race is white. Two relevant characteristics of this group were alcoholism and smoking. Therefore, early diagnosis is important to ensure the pacient's quality of life, as well as reducing the time and expenses with treatment that includes surgery and radiotherapy.

Keywords: Larynx; Neoplasm; Epidemiological
\end{abstract}

\section{Introdução}

Considerando as neoplasias que interferem na qualidade de vida de paciente em sua maioria de sexo masculino $^{1,2}$, as neoplasias malignas de laringe (NML) são um dos tipos mais comuns, cerca de $25 \%$ na região da cabeça-pescoço. São estimados 7.650 novos casos no Brasil atualmente. A laringe é dividida em supraglote, subglote e glote, que é, nas cordas vocais verdadeiras, a parte em que há maior incidência dos casos. Além disso, o carcinoma de células escamosas é o tipo histológico mais prevalente, passando de $90 \%$ dos diagnósticos ${ }^{3}$. Trata-se de um problema que apresenta variantes, como de células papilares ou fusiformes. Há ainda o carcinoma acantolítico, basalóide, papilar, verrucoso, adenoescamoso. As displasias, por sua vez, são lesões precursoras, agora, separadas em baixo e alto grau, de acordo com a arquitetura e citologia.

Tendo em vista os sinais e sintomas, se o tumor está localizado na região das cordas vocais, a rouquidão 
e sensação de bolo persistente na garganta precisam ser avaliadas por um otorrinolaringologista ou cirurgião de cabeça e pescoço. Se a tumoração não estiver próxima a essa parte, a detecção acontece mais tardiamente pelos sinais serem inespecíficos inicialmente, como tosse persistente, que pode evoluir para dispneia, dificuldade de deglutição e perda de peso. ${ }^{5} \mathrm{O}$ diagnóstico é feito por laringoscopia com retirada de amostra para exame histopatológico ${ }^{6,7}$. A maioria das amostras se encontra no estágio III ou IV, quando, geralmente, há a necessidade de cirurgia e radioterapia. $\mathrm{O}$ resultado das amostras está associado, normalmente, ao abuso de álcool, ao tabagismo e também ao Papiloma Vírus Humano (HPV), à doença do refluxo gastroesofágico, a diabetes mellitus, à hipertensão arterial e a outras doenças crônicas ${ }^{8,9,4}$. Segundo Assis $(2019)^{6}$, o tempo entre o diagnóstico e a início do tratamento foi de 44 dias, em média. A adesão ao tratamento é fundamental devido à importância do órgão para a comunicação, deglutição e estética da região do paciente. Reitera-se que a melhor forma de evitar esses danos é a partir do diagnóstico precoce ${ }^{4}$. O objetivo dessa análise é avaliar o cenário das neoplasias malignas de laringe, no estado do Rio de Janeiro, no ano de 2019, relacionando dados importantes, como o número de internações e a taxa de mortalidade em relação aos pacientes acometidos.

\section{Material e Métodos}

Foi realizada uma revisão de literatura do período de 2011 a 2020, sendo pesquisada nas bases de dados Scielo, Pubmed, Lilacs e Google Acadêmico, das quais utilizou-se as seguintes palavras para pesquisa, segundo o DeCS (Descritores em Saúde): neoplasia, laringe, epidemiologia. Associado a um levantamento de dados disponíveis no DATASUS - Sistema de Informações Hospitalares do SUS (SIH/SIS) do Ministério da Saúde (Figura1) avaliados, no período de janeiro de 2019 a dezembro de 2019, as internações, ano de atendimento, faixa etária, sexo, etnia, total de óbitos e valor total descritos em números totais e porcentagem, sendo estes presentes dados disponibilizados em tabelas no aplicativo TabNET, que serviram para subsidiar análises objetivas da situação e tomadas de decisão baseadas em evidências.

\section{Resultados}

No Brasil, de acordo com o INCA, a estimativa de novos casos da doença seria próxima de $7.650^{3}$. De acordo com os dados pesquisados fornecidos pelo Datasus (Figura 1), no estado do Rio de Janeiro, foram internadas 610 pessoas com diagnóstico de neoplasia de laringe - independentemente do tipo - no período de Janeiro de 2019 a dezembro de 2019, com gasto

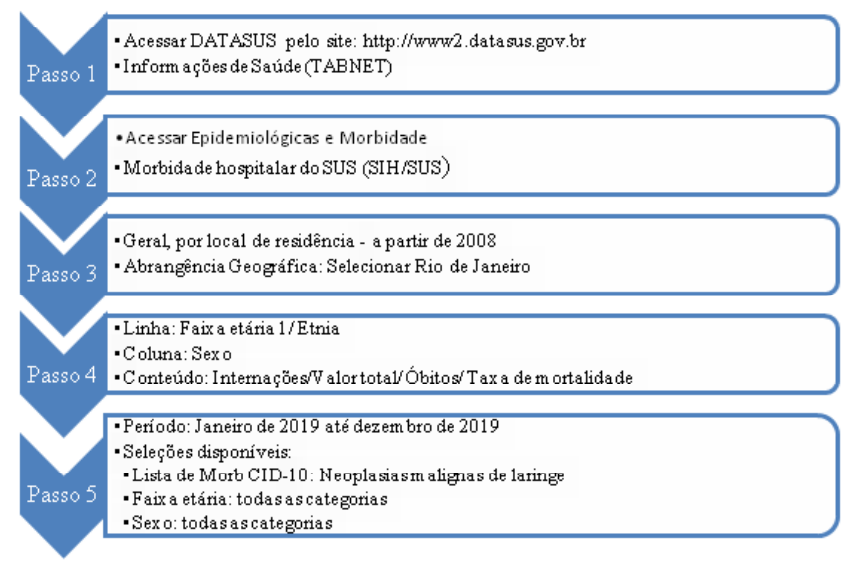

Figura 1. Fluxograma de acesso ao DATASUS.

total de $\mathrm{R} \$ 941.800,38$ e com taxa de mortalidade de 15,41 (Tabela 1). Além dessas informações, foi possível observar que a faixa etária mais afetada foi de 60 a 69 anos - com 234 pacientes (38\%), seguida da população até 59 anos - com 219 (35\%); por último, encontra-se o grupo com idade a partir dos 70 anos - com 157 (25\%). Ao analisar o sexo, temos o masculino em maior número de afetados, 518 casos totais $(84,9 \%)$. As NML foram menos prevalentes no sexo feminino, o qual apresenta total de 92 casos registrados $(15,1 \%)$ (descrito na tabela $2)^{1,8}$. Quanto às etnias, nos brancos e pardos, somam-se 336 casos (55\%). Em 191 casos, não houve informação quanto à etnia $(31,3 \%)$. O número de óbitos pela doença foi de 94 pacientes $(15,40 \%)$, em 2019, no Estado do Rio de Janeiro". Os pacientes eram, na maior parte,

Tabela 1. Internações segundo faixa etária. Lista Morb CID-10: Neoplasias malignas de laringe Período: Jan/2019 - Dez/2019

\begin{tabular}{c|c}
\hline Faixa Etária & Internações \\
\hline TOTAL & $\mathbf{6 1 0}$ \\
\hline 1 a 4 anos & 1 \\
\hline 30 a 39 anos & 8 \\
\hline 40 a 49 anos & 46 \\
\hline 50 a 59 anos & 164 \\
\hline 60 a 69 anos & 234 \\
\hline 70 a 79 anos & 126 \\
\hline 80 anos ou mais & 31 \\
\hline
\end{tabular}

Fonte: Ministério da Saúde - Sistema de Informações Hospitalares do SUS (SIH/SUS) ${ }^{8}$. 
casados, trabalhadores braçais. Apresentavam baixo nível escolar e tinham o SUS como assistência médica prioritária.

\section{Discussão}

Proteção da via aérea inferior, respiração e emissão de sons são funções exercidas pela laringe ${ }^{17}$. As neoplasias atuam causando alteração da qualidade na emissão da voz (rouquidão), disfagia leve, sensação de bolo na garganta e dispneia ${ }^{3}$. Os pacientes diagnosticados com um tipo de NML necessitam de acompanhamento médico e multidisciplinar em todas as fases, a fim de reduzir complicações psicológicas e funcionais causadas pela possibilidade de perda do órgão. Como avaliado nos resultados obtidos na pesquisa e na literatura, o perfil epidemiológico da doença é de maior incidência no sexo masculino, nos brancos e pardos, com faixa etária predominante de 60 a 79 anos, com hábito etílico e tabagismo - tanto ativo quanto passivo - em $70 \%$ a $90 \%$ dos $\operatorname{casos}^{15}$.

Deve-se considerar, ainda, que a população feminina tem adquirido tais fatores modificáveis e, como consequência disso, tem aumentado a incidência de NML nesse grupo ${ }^{11}$. Ainda foi observado que o estadiamento do câncer acontece em fases mais avançadas, e os exames de importância no diagnóstico são a laringoscopia, tomografia computadorizada ${ }^{8,12}$ e PET/CT, para detecção de metástases, além da endoscopia digestiva alta ${ }^{15}$. Como resultado disso, os casos de detecção precoce equivalentes ao T1 e T2 são tratados com radioterapia local, primeiramente, tentando preservar a laringe e, em alguns casos, cirurgia ${ }^{16}$. A maioria dos tipos avançados, equivalente a $\mathrm{T} 3$ e $\mathrm{T} 4^{16}$, tem como tratamento a cirurgia de esvaziamento cervical com traqueostomia temporária ou definitiva, associada à quimioterapia e à radioterapia ${ }^{12}$. A microcirurgia a laser transoral tornou-se popular recentemente. Ela envolve a remoção gradativa do câncer com avaliação das margens, principalmente nos cânceres glóticos e supraglóticos ${ }^{15}$. Segundo Haussman (2019) ${ }^{13}$ as técnicas de cura são perigosas e dispendiosas e, em muitos casos, não trazem ao paciente a qualidade de vida esperada.

\section{Conclusão}

Especificamente os tumores de laringe são os tipos que apresentam maior desafio de controle e conservação da estrutura devido à demora na manifestação de sintomas. Isso resulta em um diagnóstico tardio, o que, por sua vez, exige tratamentos cirúrgicos, acompanhados ou não de radioterapia, com valores de gastos totais de internação bastante elevados. Nessa patologia, observamos um perfil masculino, idoso, branco, tabagista e etilista, secundário às doenças crônicas. Não podemos deixar de considerar os casos femininos, que ainda são baixos, mas que estão em ascensão devido ao padrão da doença ser diretamente ligado ao estilo de vida. São imprescindíveis o diagnóstico precoce, as campanhas de conscientização sobre melhores hábitos de vida e as visitas regulares ao médico otorrinolaringologista.

\section{Referências}

1. Maciel CTV, Leite ICG, Soares RC, Campos RJD. Análise da qualidade de vida dos pacientes com câncer de laringe em hospital de referência na região Sudeste do Brasil. Rev. CEFAC.2 013; 15(4): 932-940.

2. Fasunla AJ, Ogundoyin AO, Onakoya PA, Nwaorgu OG. Malignant tumors of the larynx: Clinicopathologic profile and implication for late disease presentation. Nig Med J. 2016;57(5):280-285.

3. INCA. Câncer de Laringe. Disponível em: https://www.inca.gov.br/ tipos-de-cancer/cancer-de-laringe. Acesso em: 02 Ago 2020.

4. American Cancer Society. Signs and symptoms of laryngeal and hypopharyngeal cancers. Disponível em: https://www.cancer.org/cancer/ laryngeal-and-hypopharyngeal-cancer/detection-diagnosis-staging/signssymptoms.html Acesso em: 02 Ago 2020.

5. Santana-Álvarez J, Acosta-Abréu GB, Miranda-Ramos MÁ, LeónMolina M, Quiroga-Meriño E. Eficacia de la radioterapia em los pacientes com cáncer laríngeo en estadio I y II. AMC 2018;22(6):725-742.

6. Davaris N, Voigt-Zimmermann S, Kropf S et al. Flexible transnasal endoscopy with white light or narrow band imaging for the diagnosis of laryngeal malignancy: diagnostic value, observer variability and influence of previous laryngeal surgery. Eur Arch Otorhinolaryngol 2019; 276:459-466. Disponível em: https://doi.org/10.1007/s00405-018-5256-1. Acesso em: 02 ago 2020 .

7. Assis, JLP. Tempo até o tratamento de pacientes com câncer de cabeça e pescoço no estado de São Paulo: 2011 a 2017. São Paulo: Universidade de São Paulo, Faculdade de saúde pública. 2019. Disponível em: https://doi. org/10.11606/D.6.2019.tde-26082019-130954. Acesso: 02 ago 2020

8. Dedivitis RA, Tincani AJ, Chone CT, Cernea CR, Montenegro FLM, Hojaij FC et al. Câncer de Laringe: Diagnóstico. Projeto Diretrizes Associação Médica Brasileira e Conselho Federal de Medicina. 2011. 1-16. Disponível em: https://diretrizes.amb.org.br/_BibliotecaAntiga/cancer_de laringe diagnostico.pdf. Acesso 2 ago 2020

9. Reicher Y, Alkan U, Levi L, Bachar G, Popovtzer A. Prognostic factors for survival and nonfunctional larynx in patients with squamous cell carcinoma of the larynx. The Laringoscope 2020;130: 1202-1205.

10. DATASUS (SIH-SUS) - avaliado de jan de 2019 a dez de 2019, avaliando geral por local de residência, internações, ano de atendimento, taxa de mortalidade, óbitos, faixa etária, sexo, etnia e valor total. Acesso em: 02 de ago 2020.

11. Pereira IF, Noronha VRAS, Naves MD, Amaral TMP, Santos VR. Neoplasias malignas em região de cabeça e pescoço: perfil dos pacientes atendidos na UFMG. Rev Cub Estomatol 2019; 53: 233-244.

12. García-León FJ, García-Estepa R, Romero-Tabares A, Gómez-Millán Borrachina J. Treatment of advanced laryngeal cancer and quality of life. Systematic review. Acta Otorrinolaringol Esp. 2017;68(4):212-219.

13. Hausman DM. What Is Cancer? Perspect Biol Med. 2019;62(4):778784.

14. Fernandes GM, Bergmann A, Oliveira JF. Análise epidemiológica de população com câncer de cabeça e pescoço: influência sobre as complicações pós-operatórias Rev Bras Cir Cabeça Pescoço. 2013;42(3): 140-149.

15. Koroulakis A, Agarwal M. Laryngeal Cancer. StatPearls Publishing [Internet]; 2021 Jan. [Acesso 06 abril 2021]: Disponível: https://www.ncbi. nlm.nih.gov/books/NBK526076. 
16. Pfister DG, Laurie AS, Weinstein GS, Mendenhall WM et al.

American Society of Clinical Oncology Clinical Practice Guideline for the

Use of Larynx-Preservation Strategies in the Treatment of Laryngeal Cancer.

$\mathrm{J}$ Clin Oncol 2016;24(22):3693-3704.

17. Padial MB, Ronchi DI, Madeira K. Perfil epidemiológico das neoplasias malignas da laringe em um laboratório de anatomia patológica de

Criciúma - SC no período de 2006 a 2010. Arq Cat Med 2011;40(4):64-68. 